Open Ocean • Here be Dragons 2018

\title{
Ocean Cultures Report
}

Devora Najjar ${ }^{1}$ Avery Normandin ${ }^{1}$

${ }^{1}$ MIT Media Lab

Published on: Sep 30, 2021

DOI: https://doi.org/10.21428/a680be9a.207c346e/e79271f1

License: Creative Commons Attribution 4.0 International License (CC-BY 4.0). 


\section{Goals}

Like many indigenous groups, the Māori of Aotearoa (New Zealand) have a long and deep connection to their marine ecosystems, with rich culture and communal practices on responsible management of their waters. With culturally relevant fish stocks now threatened due to overfishing and ecological changes, increasing tools for communities to use for marine monitoring could help indigenous communities with local management of their waters. Ocean Cultures focused on co-developing and implementing sensing systems within the Horokaka and Toka Tāmure Mataitai, protected areas of ocean on the Mahia Peninsula where Maori locals manage all noncommercial fishing. The data generated through sensing will contribute to documentation of the mataitai with the goal of identifying appropriate sites for a species transplantation program for abalone no longer found in the mataitai. Additionally, this program planned to support educational efforts led by Maori elders within the community that teach traditional forms of ecological monitoring. Together, this monitoring program can allow for community members to have a holistic understanding of their ocean health.

\section{Approach}

The key goal of the program was to integrate data acquired both by sensor technologies as well as indigenous cultural practices as a platform for community-led marine monitoring and management. A more cohesive synthesis between results in a more vibrant portrait of ecosystem health and offers communities routes to intervene accordingly. To do this, we participated in a series of wānanga, a Māori method of generating and sharing knowledge, in order to discuss the current state of sensor system and generate diverse community involvement and input through traditional methods.

Over the course of our time in Mahia, topics discussed included:

1. How to design a framework for community-led marine sensor technology that prioritizes their values, goals, and vision for their marine ecosystem;

2. Methods of storing and analyzing the data that are generated and accessible locally within the Mahia community;

3. Planning future wānanga for all ages on sensor systems for monitoring, as well as traditional monitoring techniques that will be open to the entire Mahia community to question, learn, and modify these methods moving forward; and,

4. Potential timeline for baseline ecosystem data collection with the intention of using this information to design a species reintroduction program for paua (abalone) from the peninsula that are no longer found in the waters on the east coast.

We also spent a significant amount of time on the reefs together with community members which allowed for contextualization and further discussions on monitoring efforts. While on the reefs we focused on wave 
dynamics, tidal conditions, sedimentation, seaweed populations, and possible locations of interest for further monitoring and future transplanting.

\section{Accomplishments}

The main accomplishment of this trip was in establishing trust within the community and learning more about their goals for the monitoring of their mataitai. We attended community discussions that focused on the tangata whenua's long history on the Mahia peninsula and the ways that the peninsula's beaches and intertidal waters have been used over time. Though the mataitai had developed comprehensive documents about their goals for the mataitai, there was information that we learned through these meetings which provided a much fuller picture of the community goals. Specifically, we learned more about the community's current monitoring program and technical capacity with respect to both transect- and scuba-based approaches, as well as providing information on larger watershed-related concerns due to changing land use which has caused increased sedimentation within the intertidal zone in recent years.

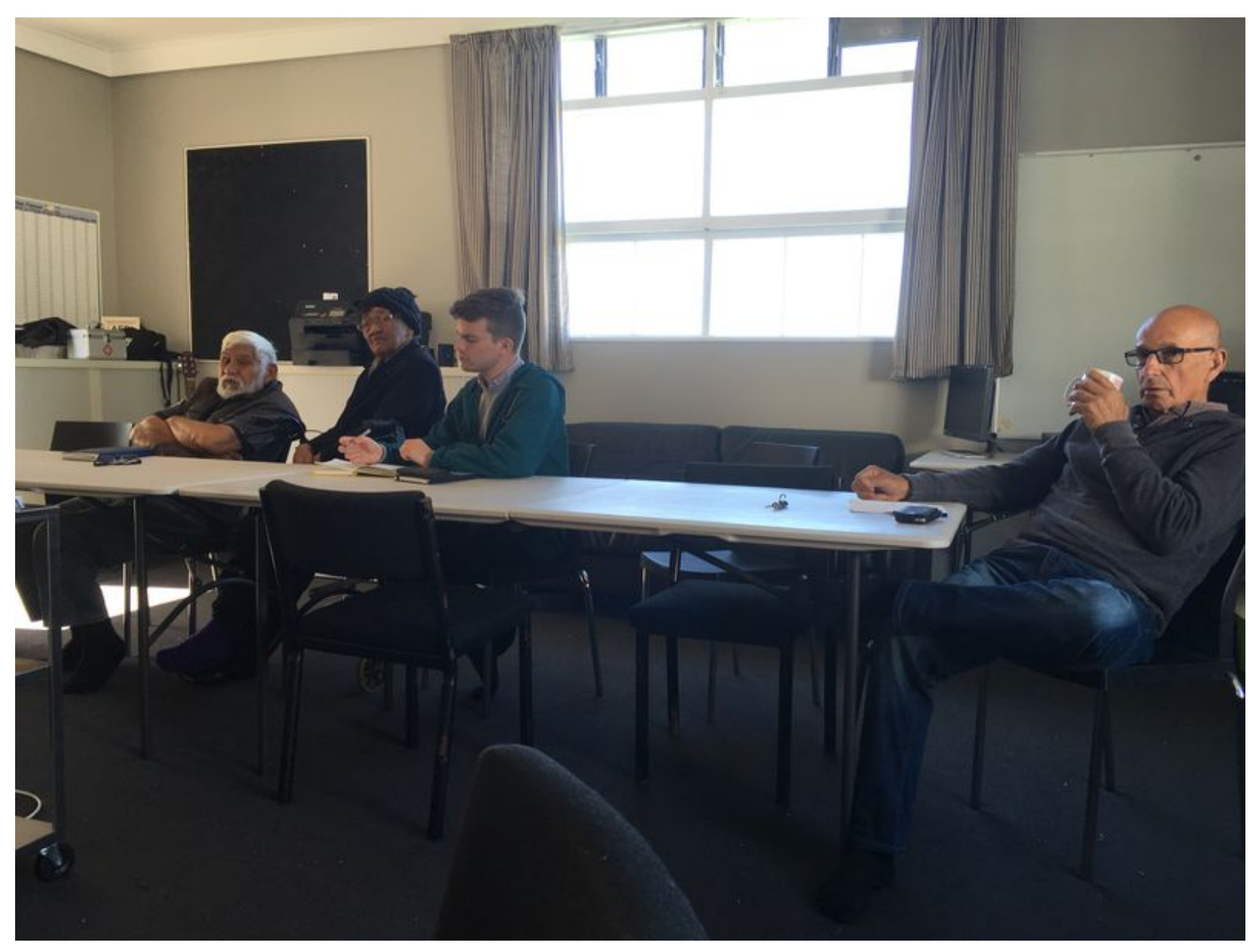




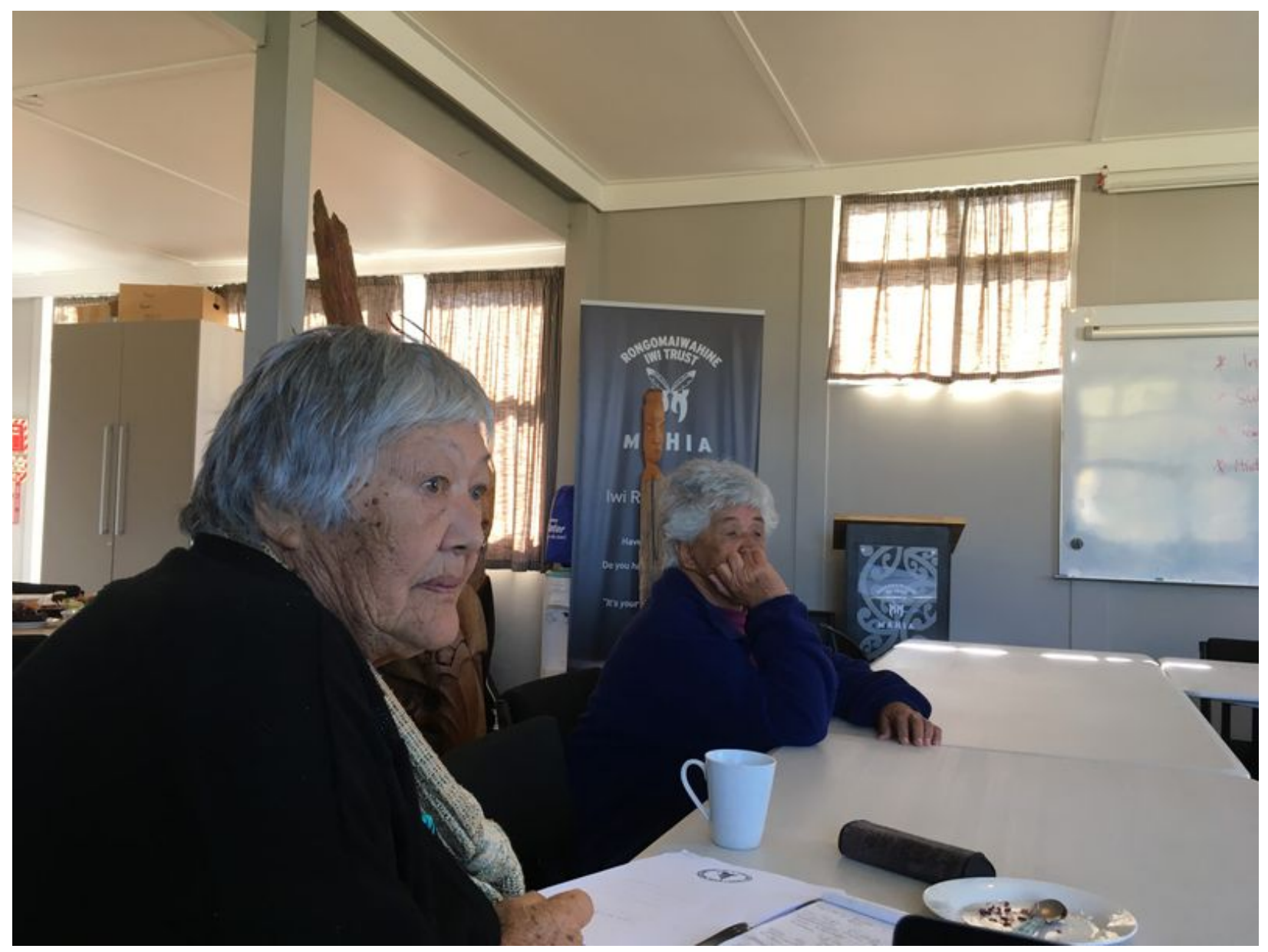




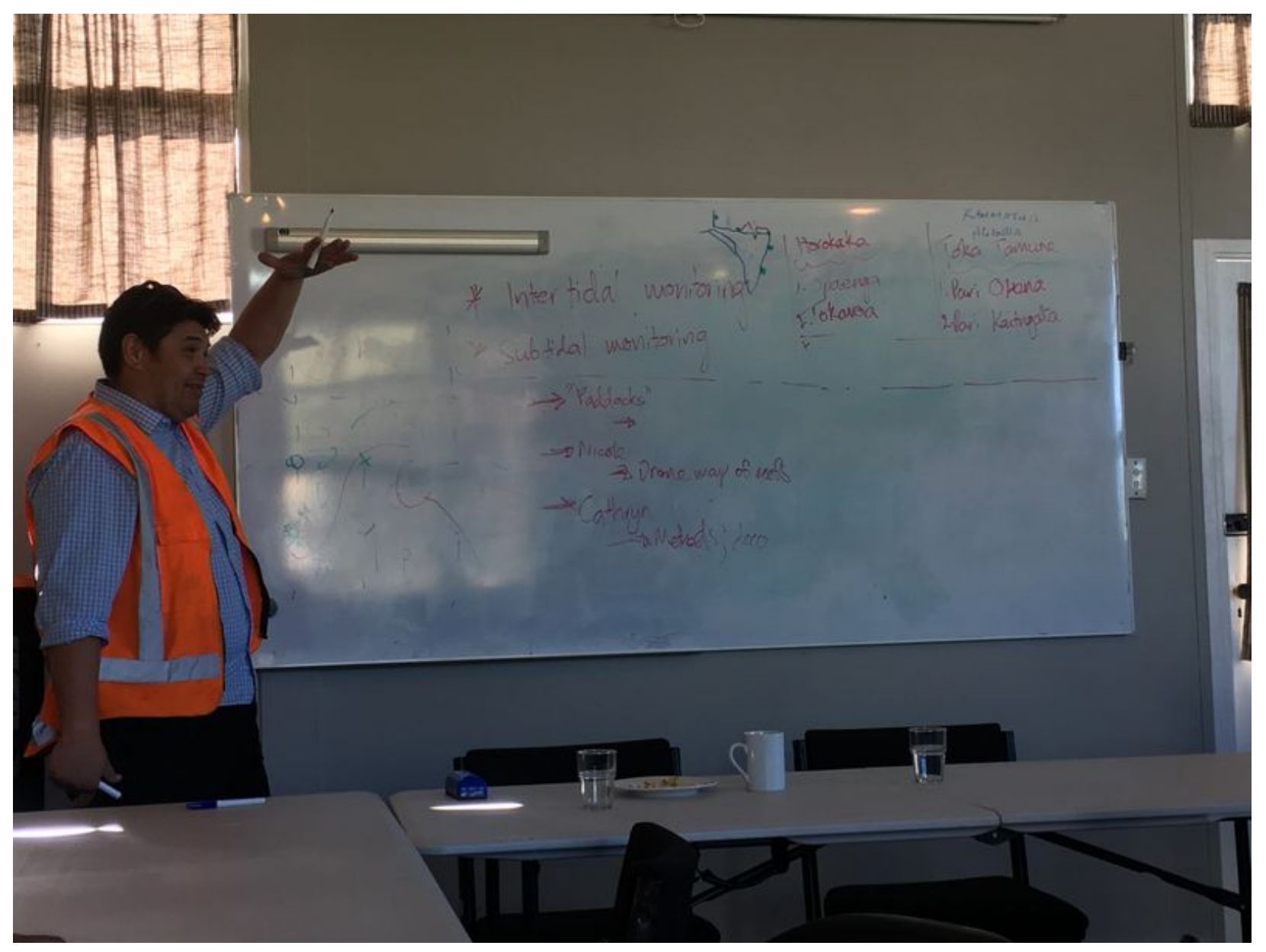




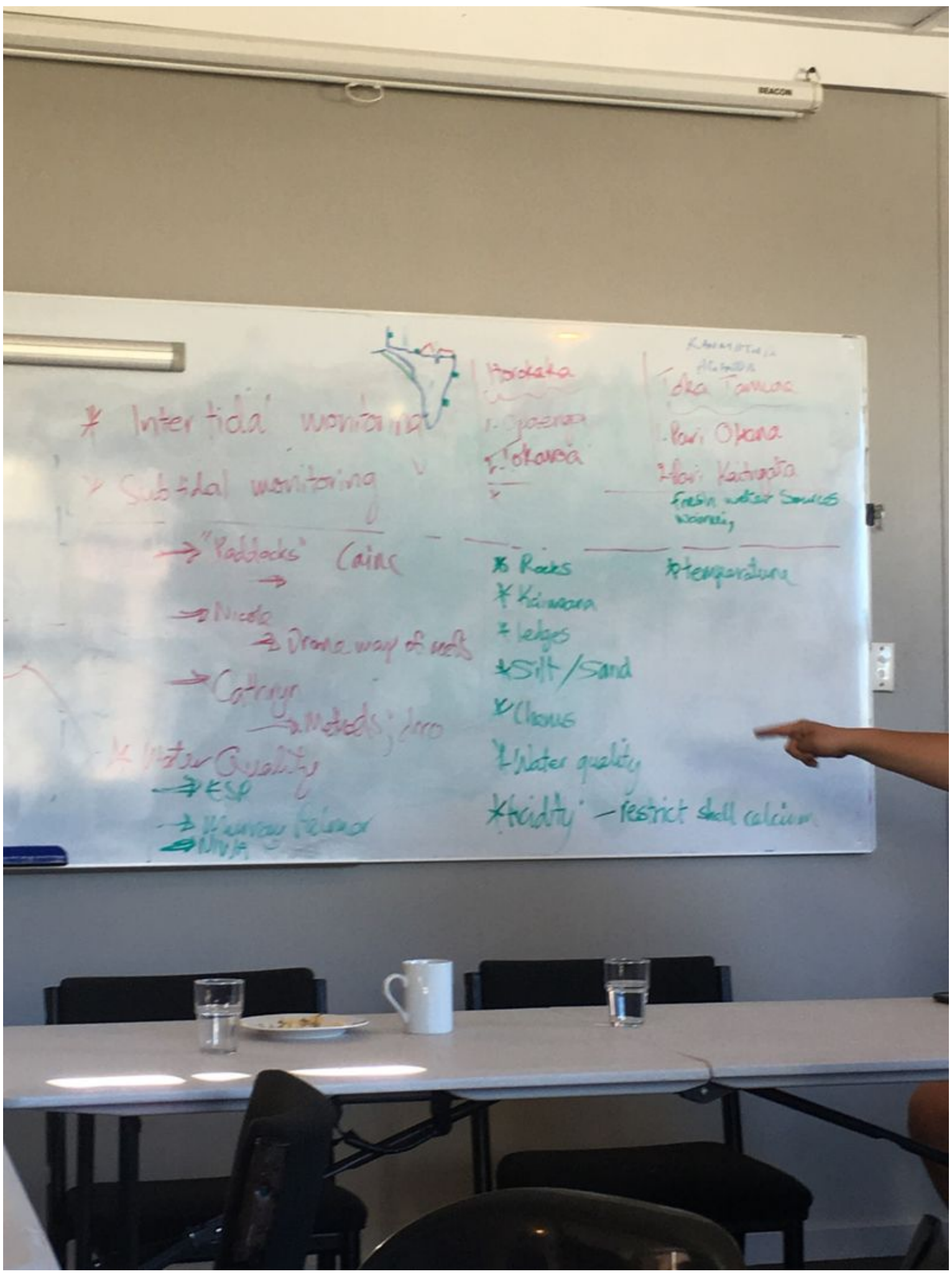

Similarly, meetings with individual community members allowed us to gain a better understanding of individual goals and interests, as well as to learn more about history and life on the peninsula, as well as a 
chance to better understand local and regional politics which could impact the mataitai and programs overall.

Overall, this trip allowed for a strong foundation for collaboration, as well as provided a clearer picture of the monitoring needs for the community, as well as sensors that would be potentially useful.

\section{Challenges}

The main challenge associated with this project was realizing that things are never exactly as they appear! When first connected with the community and documentations about the mataitai, it seems that the whole program was set and we would arrive to listen, learn, and discuss additional monitoring technologies. Upon arriving and learning more, it was clear that the monitoring program, while in progress, was a living thing. This means that all of the baseline monitoring which was assumed at the outset was not necessarily set in stone. This made the trip more challenging, but ultimately all the more worthwhile because we were able to better understand the community's desired for the data and outcomes of the monitoring better.

\section{Next steps}

July 2019 (grant through the PKG Fellowship)

While in Mahia, I worked closely with community members through a series of community meetings, or wānanga, to introduce the monitoring systems to support the health of the marine ecosystem. The goal was for this to be an iterative system, so the monitoring systems that were brought and tested were the first in a series of prototyping activities that the community will participate in to assure that all opinions are heard. The sensors that were part of the first iteration included both analytical-grade probes for $\mathrm{pH}$, dissolved oxygen, temperature, and conductivity, as well as experimental sensors that were attached to LEDs and changed color in response to changing water quality. The community sessions were planned as wānanga that took place in the local marae, which allowed for the local community to control the meeting and provided a space where a diversity of perspectives could be present during the process (we even had a member of the regional council and a department of conservation employee attend!).

Wānanga \#1: This workshop was focused on understanding the history of the tangata whenua on the peninsula, as well as using the sensors that were designed. The morning was opened by the local elders, who spent time speaking on their childhoods on the peninsula, stories from their parents and grandparents on the history of the peninsula and their connection, as well as changes that they've seen to the intertidal zone. Following lunch, we regrouped to discuss the water sensors and planned the areas where we would go to collect water based on the morning's discussion. After community members collected water, we analyzed and discussed the data collected. This provided a strong place-based foundation for the monitoring, a hands-on activity in collecting and processing water quality data, and space to collectively analyze and discuss the data generated. 
Wānanga \#2: This workshop was also held at the marae and was focused on discussing data management and analysis methods for the sensor data generated. We first debriefed about the first wānanga and changes that can be made for the future. We then had a brainstorming session on areas of the mataitai that were best suited for frequent monitoring with the sensors, as well as as well as monitoring methods for potential transplanted species.

\section{Acknowledgements}

Ocean Cultures was funded by the MIT Media Lab Open Ocean Initiative, MIT PKG Center, and National Geographic Society. 